\title{
Training in the new driving and failures simulator of Metro de Madrid (SICAMM)
}

\author{
J. M. Mera, L. M. Gutiérrez, E. Castellote \& G. Corcho \\ CITEF (Railway Technologies Research Centre), \\ Escuela Técnica Superior de Ingenieros Industriales, \\ Universidad Politécnica de Madrid, José Gutierrez Abascal, Madrid, \\ Spain
}

\begin{abstract}
The new Metro de Madrid Driving and Failures Simulator represents the physical realization of the main ideas when it comes to development aimed at driver training.

The simulator consists of a scale model of a car with real equipment integrated into the simulation. All the equipment and/or controls comprising the simulated series (7000 and 8000 ) are reproduced to allow training in an immersive environment with a four channel visual system.

Also, a set of virtual simulators is integrated into the full scale simulator and they are able to interact with the cab to do manoeuvres exercises, or to do independent training exercises. In this way a two step virtual training tool is available, wholly integrated into a single Training Centre and controlled by one Instructor.
\end{abstract}

Among the capacities with which the set of simulators is endowed, not only is the reproduction of the whole train emphasized but also the capacity to do training exercises with train failures (approximately 500 different ones) or incidents (approximately 50 different types).

The developed model allows the status of the different electrical and pneumatic circuits making up the trains to be displayed. This allows the students to know the internal operation of the train and to understand the behaviour of the vehicles in the different failures that can be displayed.

Also, joint exercises between training positions are allowed to practice coupling and de-coupling between train units.

The infrastructure reproduced comprises four of the most modern lines of Metro de Madrid (8, 9, 10 and 12), including the different field elements (signals, switches, interlocking ...).

The simulated Control Centre position allows drivers and signalmen to be trained together. The user interface has been designed with a user interface similar to that existing in the Control Centre. This feature increases training possibilities for personnel.

The control level on the simulator enables a centralized database for the exercises done by each student to be kept, and training plans to be prepared, as well as to display and manage the completion of these training plans.

All these elements together reach a new level in driver and operator training for metros.

Keywords: driver training, system simulation, high immersive training. 


\section{Objectives and state of the art}

In the period 1999-2003, Metro de Madrid faced one of the greatest challenges in its history, the largest Infrastructure Extension Plan embarked upon by the company since its foundation.

This plan aimed at building $54.3 \mathrm{~km}$ of new tunnels on three different lines (to obtain a total of $226.9 \mathrm{~km}$ ) in a period of four years, in addition to updating its fleet of trains with 84 new units with two new 7000 and 8000 series (making a total of 265 trains).

Table 1: New sections in Extension Plan (19992003).

\begin{tabular}{|l|l|l|}
\hline Line & Length & Stations \\
\hline Line 8 & $5,85 \mathrm{~km}$ & $\mathbf{2}$ \\
\hline Line 10 & $7,70 \mathrm{~km}$ & $\mathbf{6}$ \\
\hline Line 12 & $40,75 \mathrm{~km}$ & $\mathbf{2 8}$ \\
\hline Total & $54.3 \mathrm{~km}$ & 36 \\
\hline
\end{tabular}

Table 2: New rolling stock for Extension Plan (1999-2003).

\begin{tabular}{|l|l|l|}
\hline Train class & Units & Cars \\
\hline 6000 (non engine car) & - & $\mathbf{3 5}$ \\
\hline 8000 series $(1500 \mathrm{~V})$ & 37 & $\mathbf{1 1 1}$ \\
\hline $\begin{array}{l}8000 \text { series (dual } \\
\text { voltage) }\end{array}$ & 10 & $\mathbf{3 0}$ \\
\hline 7000 series (1500 V) & 30 & $\mathbf{1 8 0}$ \\
\hline $\begin{array}{l}7000 \text { series (dual } \\
\text { voltage) }\end{array}$ & 7 & $\mathbf{4 2}$ \\
\hline Total & 84 & 398 \\
\hline
\end{tabular}

The consequences of embarking upon an extension plan of this magnitude in a short period of time are the high staffing requirements needed to implement such plans, as well as the short period of time available for training. In addition, the staff training plans had to be modified so that the challenge could be met by accepting as a basic premise that most of the training would be given without having the availability of either the new infrastructures or the new trains.

In order to meet the aims previously defined, solutions adopted by others companies were analysed. The first conclusion was that the new training plans would have to use new rolling stock simulators with virtual reproductions of the new infrastructures so that training could go ahead without any need to have access to the new railway infrastructure or trains.

Moreover, these simulators would be available before the real systems so that they could meet one of the basic aims, that is facilitate the implementation of the new material resources according to specific schedules.

After that decision, an analysis of the state of the art of training simulators in railways was made to know the appropriate characteristics for the simulator. The analysis shows that there are three simulator levels:

1. Limited environment with real controls on occasions, and small projection systems (mini-simulators) [2,10].

2. Environments with real controls and relatively large projection systems $[2,3,7]$.

3. Immersive environments with moving platform systems $[4,5,8]$. 
As the level increases, the elements that increase the sensation of being totally immersed in a real environment also increase. However, although each simulation project has specific features, the majority have some general features in common:

- Design oriented towards driving in non-degraded and degraded conditions with a set of the most frequent failures (around 20-30) $[8,10,12]$.

- Design oriented towards reproducing driver sensations, which is achieved by an environmental display based on showing appearances: signal status, traffic...[4,5].

- Driver's desk reproduction hardware, the other train elements being reproduced on very few occasions $[2,4,5]$.

- Driving from a cab $[2-5,7,8]$.

- Simulator control from a specific post with student-instructor relations (in some cases user supervision is not required) $[2-5,7,8]$.

Nevertheless, in order to allow the use of the new materials to be explored in greater depth, Metro de Madrid's training needs include specific problems:

- To improve drivers' knowledge of how the train works, as well as the elements that can be activated by driving personnel throughout the train unit.

- To improve fault solution time, in a very wide number of faults.

- To overcome communication problems between driving personnel and the control centre.

- To provide training in the new elements installed in trains such as the evacuation stairs and the SDPI system (fire extinction).

Most of these elements and situations cannot be trained with the common characteristics, therefore the new Metro de Madrid's simulator needed to redefine some to adapt it for the new trainings objectives and to perform research and development to have some others available as they did not exist in the market at that time.

\section{Concept of the simulator for Metro de Madrid}

Once training needs have been defined, and the training procedures used by other administrations have been analyzed, the traditional concept of a Driving Simulator is redefined to change it into a training Centre.

Two training Centres are set up, the first is located in Canillejas and devoted to training in 8000 series rolling stock, and the Cuatro Vientos Centre devoted to 7000 series training. Both Centres, however, are capable of giving training in both types of rolling stock since the elements that can be activated in both series are fairly similar.

Each of the Training Centres has the same features:

- A training Centre Control Post called the Instructor's Post, which is also responsible for planning training. 


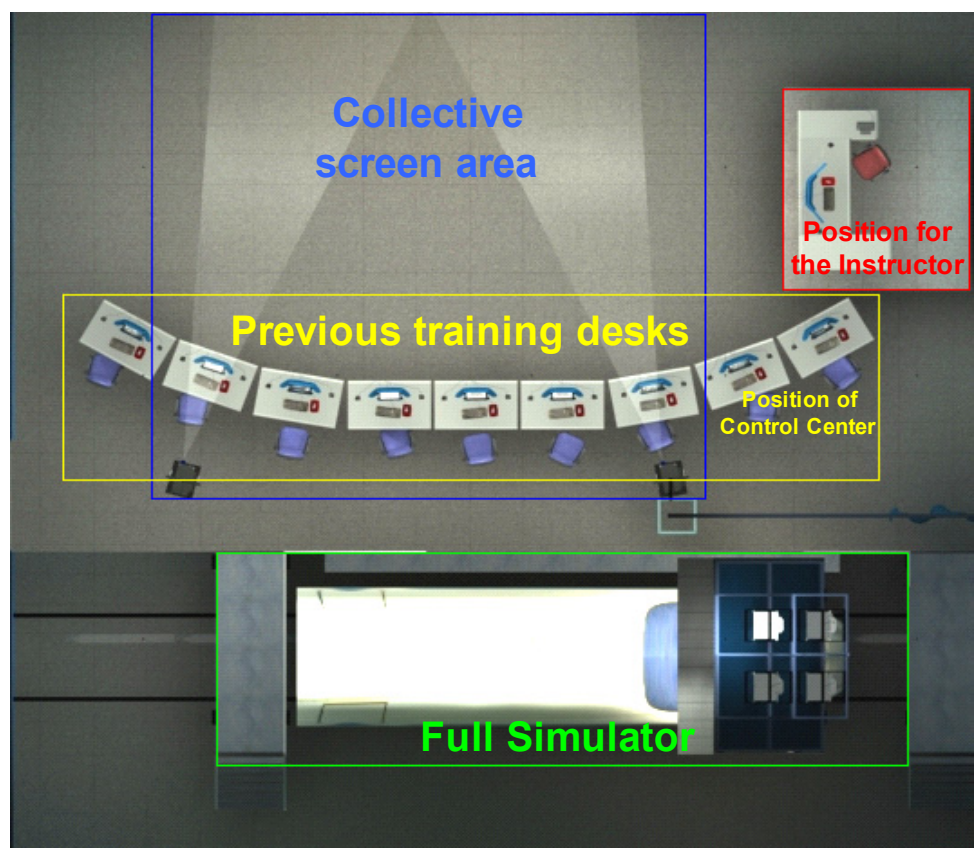

Figure 1: Elements distribution in the Training Centre.

- $\quad$ Eight training posts made up of computer equipment.

- A training post installed on a model of a real train.

- A collective projection system whereby students can see images of the instructor's post.

\section{Training centre resources}

\subsection{Instructor's position (PI)}

This is the central control desk of the Training Centre. The Instructor can use a management tool that allows:

- Creation of exercises for the simulator and learning test.

- Definition of lessons and training plans for the students.

- User and training plan management (definition of exercises to be made by the students).

- Supervision of the completion of exercises in the real scale model.

- Modification of the conditions of completion of exercises in the real scale model: introduction of failures or incidents, changes in the traffic, management of the infrastructure, changes in the status of controls ...

- Analysis of the exercises done by drivers (to see the status of the train; to see the visual system, to hear sounds from the cab; to see the closed circuit TV ...). 
Due to the fact that all the tasks that can be performed by an instructor at the same time, the desk uses four monitors (two for the control program, another for the visual system and another for the closed circuit TV).

\subsection{Full simulator}

This is installed on a full scale model of a car of about eleven meters with its real equipment. All the controls that can be found in the simulated series (7000 and 8000 ) are reproduced by real elements to obtain an immersive environment. The objective is for the driver to be unable to perceive any differences between a real train and the scale model.

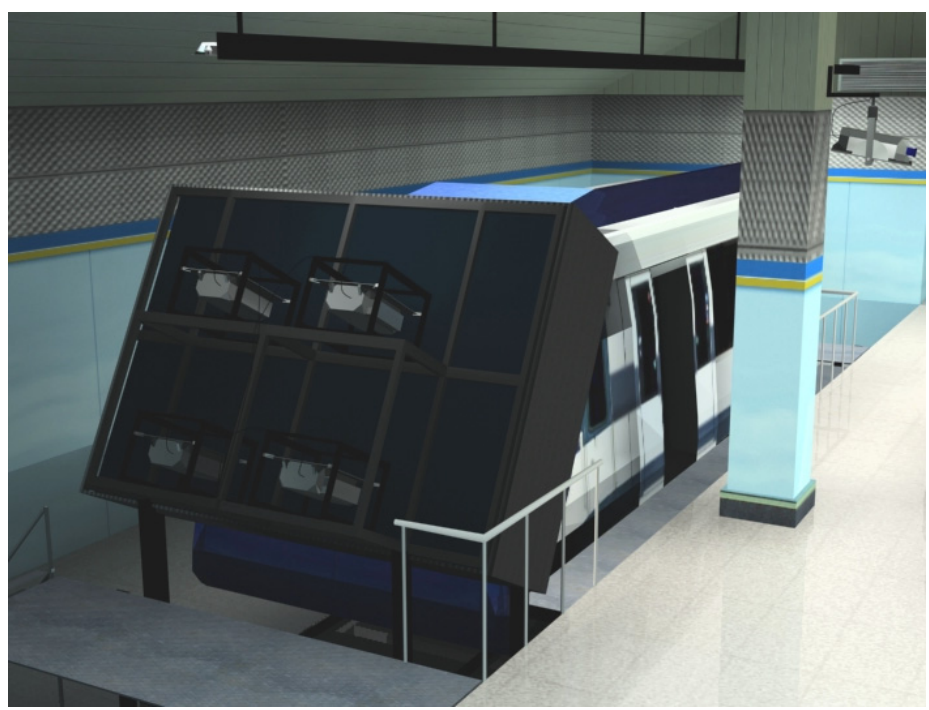

Figure 2: The scale model with the projection system.

All the elements located outside the eleven meters of the scale model are represented by a virtual switch panel system. These virtual switch panels are compatible with the status of the elements in the real panels and the same virtual representation is employed in the PI.

All the previous elements are integrated into the simulation models that are used during the simulation exercises for training, and respond to the conditions indicated by the PI of the initial conditions for the exercise (including failures, incidents...).

Aimed at enhancing the sensation of total immersion, the simulator has been completed with a synthetic image system which consists of one screen and four independent projection channels. The system is mounted covering the window in the front of the train.

Naturally, a sound system that reproduces the sounds perceived by the driver has also been included inside the cabin (pneumatic systems, alerts sound, passenger messages, station indications...) 


\subsection{Previous training desk (PFP)}

This is the individual desk that allows the same exercises as in the scale model to be done in a completely virtual desktop environment. Each of these desks is controlled by the PI in the same way as the scale model.

Each Training Centre has eight pre-training desks, where all the train's handled elements are represented with virtual photorealistic images.

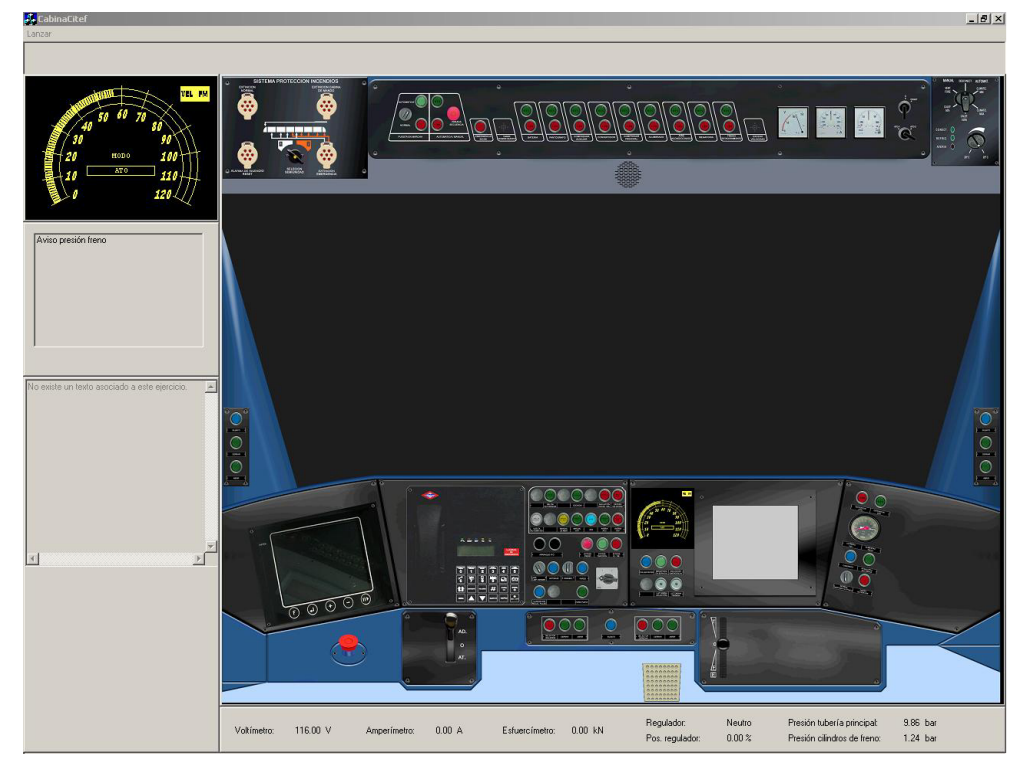

Figure 3: $\quad$ Virtual driving desk for the 7000 train.

The mathematical models used are analogous to those employed in the scale model, although they do not have the same level of exigency because they do not have a virtual display system. Each desk is able to reproduce the same exercises as in the Full Simulator (except docking and undocking exercises which require two simulators working together) but with fewer computers and therefore less quality.

With the objective of showing how the trains work, dynamic schematics of the pneumatic and electrical system can be shown. This shows how the train responds to the driver's actions.

\subsection{Collective screen}

This is a set of two independent screens with their respective projectors that are managed from the PI. Therefore it is possible to emit signals from PI, closed circuit TV or an external computer for each screen. This is used for giving lectures to several students together. 


\section{Training system capacities}

\subsection{Train modelling}

The detail of train modelling encompasses the reproduction of all elements that can be activated either directly or indirectly by the driver, thus, access is had to the different driving cabs comprising the train at any moment.

The electrical circuits are simulated on a logical level, while the pneumatic circuits take account of the dynamic air flow according to the train's circumstances.

The electronic equipment is reproduced on a logical level, and if there is user interaction on a visual level (Driving aid terminal, digital speedometer, radio console display ...).

The reproduction of the above systems allows the train to be driven on a basic level from any of its cabs, even simultaneously, as well as permitting interaction with all the elements distributed throughout the train.

There have been modelled 55 electrical and pneumatic planes per car $(6$ cars for series 7000 and 3 cars for series 8000) approximately. Each car is represented with 1.500 components, so the 7000 and 8000 series have 9.000 and 4.500 components for modelling the electric, pneumatic and electronic systems.

\subsection{Circuit reproduction}

The high level of the developed model allows the status of the different electrical and pneumatic circuits making up the trains to be displayed. This display can be seen in the PFP or in the PI during a simulation, and explains how the train works during a driving procedure or a failure, allowing the students to know the internal operation of the train and to understand the performance of the vehicles in the different failures that can be displayed.

\subsection{Fire extinction system}

The reproduction of all the train's electronic systems is particularly focused on the fire extinction system. This performance model takes on great importance from a training point of view since the system cannot be used on real trains as water and chemical compounds are emitted inside the train. Therefore, fire practice training is done solely with the simulator.

\subsection{Joint exercises}

The highly detailed train modelling is completed by the ability to reproduce manoeuvre exercises that take account of the presence of two trains.

Among these exercises, of particular interest are coupling and uncoupling units, as well as driving from the intermediate cab. These exercises allow training in towing and battery aid from another train, the most common situations to force coupling manoeuvres. 

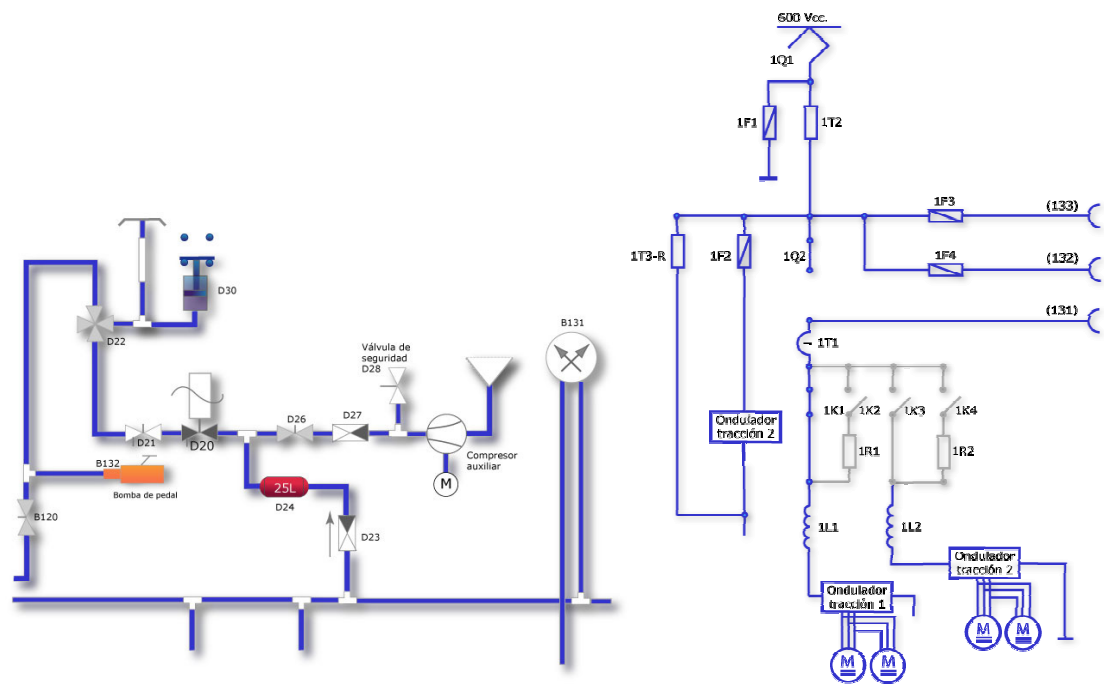

Figure 4: Visualization of a pneumatic and an electric system.

\subsection{Real infrastructure reproduction}

One important item in the training aims of the project was to obtain an immersive environment, so the project used a synthetic visual system that reproduced an infrastructure made up of four of the most modern lines of Metro de Madrid (8, 9, 10 and 12), including the different field elements (signals, switches, interlocking ...).

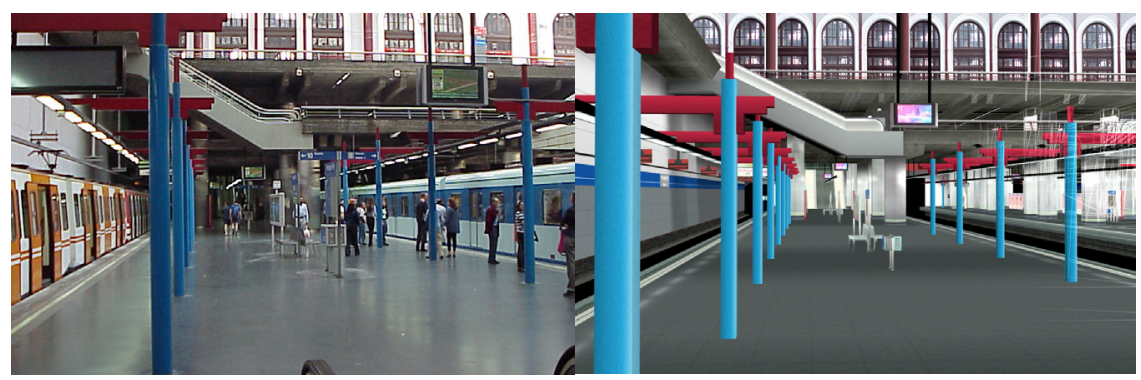

Figure 5: Comparison: photography against image from DB of the visual system for Principe Pio station.

The reasons for using these lines were that the new trains are used on these ones. But not only the operational lines are represented, but also the depots and the connections between the lines and the depots are also included. So the simulator can be used for manoeuvres of interconnection between lines and movement in depots. 
Table 3: $\quad$ Virtual scenario constructed.

\begin{tabular}{|l|l|l|l|} 
& \multicolumn{1}{|l}{ Length } & \multicolumn{1}{c|}{ Stations } & Depots \\
\hline Line 8 & $13.90 \mathrm{~km}$ & $\mathbf{6}$ & $\mathbf{0}$ \\
\hline Line 9 & $38 \mathrm{~km}$ & $\mathbf{2 2}$ & $\mathbf{2}$ \\
\hline Line 10 & $24.29 \mathrm{~km}$ & $\mathbf{1 9}$ & $\mathbf{2}$ \\
\hline Line 12 & $40.6 \mathrm{~km}$ & $\mathbf{2 8}$ & $\mathbf{2}$ \\
\hline Total & $116.79 \mathrm{~km}$ & 75 & 6 \\
\hline
\end{tabular}

\subsection{Failures and incidents}

The definition of the project for each Centre endowed the set of simulators not only with the reproduction of the entire train in normal situations, but also with the capacity to make training exercises with train failures.

Approximately 500 different failures are taken into consideration between the two rolling stocks ( 7000 series and 8000 series), in addition to 50 types of incidents for each series.

In the case of infrastructure field elements, any of the elements available can be made to fail (points, crossovers, balises, signals, derailment device...); in addition, 10 line incidents are foreseen (obstacle on the line, persons trapped, persons run over...).

All these failures and incidents can be programmed as part of an exercise so that they will happen as the train passes a certain point or at a particular time. In this way, a flexible system for generating atypical driving can be generated that is easily reproducible for a group of students.

\subsection{Infrastructure and traffic}

A simplified Control Centre has been simulated for controlling the field elements of the Infrastructure. This functionality allows the Instructor to control the signalling system or allow them to use the PI to train drivers and signalmen together. The user interface has been designed with a similar user interface to that existing in the Control Centre. This feature increases training possibilities for personnel.

The signalling system has been reproduced in depth, so the ATP and ATO onboard system (for the driving train) received the same frequency codes that a real train process in the line represented. This simulation model has been obtained representing all the elements associated with the real system (radio and balise subsystems) and analyzing how works. The ATP and ATO models include distance objective and velocity codes.

Also, the traffic of an exercise can be managed by setting the level of trains circulating in the infrastructure, as well as limiting their speed or eliminating or inserting trains. All the trains behave automatically, complying with running regulations and making stops as if they were trains in service on the line. This capacity to make simulated traffic available enables the student to perceive the consequences of their actions on the overall running of the line (separation and approach of trains, suspension of the service ...). 


\section{Training procedures}

The original training idea was to use the simulator for drivers, but due to operational and maintenance reasons, Metro de Madrid needs to use the simulator for training internal and external personnel in driving. These new students only need to know how to drive in normal situations where a professional driver is not needed.

The status display for the different electrical and pneumatic elements offers a new training possibility; the simulator can be used by engineers and maintenance personnel who need to understand how the train works.

The user interface, similar to that existing in the Control Centre, used to control the signalling system and the traffic, increases training possibilities for Control Centre personnel.

As a result, the training procedures are organized for the training of a wide variety of personnel with different objectives.

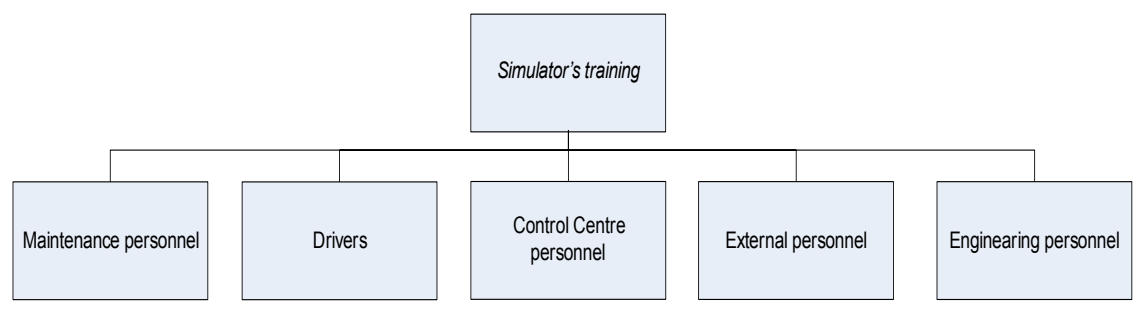

Figure 6: Personal that uses the simulator training system.

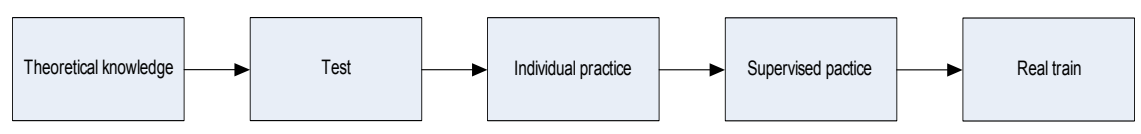

Figure 7: The five steps learning system.

The features of the Training Centre enable a training procedure to be carried out in which a student's level of training is organized in five steps.

Each one of these steps allows differentiated progress in driver training to be attained.

The different steps comprising the training procedures are:

1. Theoretical knowledge

Using the two collective screens, theoretical training classes are distributed.

The PI computer is used to make presentations, to show videos and images, as well as real-time images from the Full Simulator during an exercise. In this way, it is possible to teach different aspects of the train, as well as to show how operational procedures must be performed.

2. Consolidation of theoretical knowledge

Before doing practical exercises, a simple series of tests must be done by the students on the PFP. These tests enable the knowledge acquired from the theoretical classes to be checked. 


\section{Learning practical knowledge}

Once the theoretical knowledge has been checked, the practice starts. Using the same PFP, the student has a display of the simulated train.

On this display the student can become familiar with the normal operational procedures and the solving of failures.

In order to validate the knowledge, the students must make a training plan comprising a set of exercises. The results from all the exercises are stored in the database of the Training Centre for later analysis.

4. Consolidation of practical knowledge

Once acquisition of the necessary theoretical and practical knowledge has been demonstrated, the student can use the Full Simulator.

This step is similar to the previous one, but uses a high quality environment for training. The use of the Full Simulator means that a real train need not be used until a high level of knowledge is acquired by the students.

The student knows the equipment and the controls making up the train before using the Full Simulator, and learns how to physically manipulate it and in unexpected situations.

The scale model completed with a synthetic image system and a sound system means that an immersive environment can be obtained that increases the realism of the training. Therefore, it is possible to practice driving exercises, manoeuvres exercises (docking and undocking), as well as circulation with traffic.

The Full Simulator can be used for single and joint exercises, using one PFP and/or using the PI. In the latter case, a driver and signalman can do exercises simultaneously, thereby increasing the training experience.

Additionally, the greater equipment allows practice in the handling of other equipment from the train: radiophone, closed circuit TV... These pieces of equipment only have a mathematical model in the Full Simulator.

Finally, this post provides an additional advantage; the set is supervised by the PI continuously and the instructor can modify in real-time the exercise conditions. As in other cases, all the results from the exercises are stored in the database of the Training Centre for later analysis.

5. Practice with a real train

This is the last step in the training procedure. Once the student has acquired the necessary level of training, they are ready to use their knowledge in a real train.

\section{Conclusions}

The highly detailed modelling of the train's electrical and pneumatic circuits has enabled a training tool to be obtained that shows the behaviour of all the systems making up the trains with a high degree of realism. Moreover, it facilitates the implementation of failures since many of their aspects are a direct result of the topology and functionality of the electrical and pneumatic circuits.

The realism attained, means that the quality of training given with the simulator can be enhanced, not only in train driving but also in troubleshooting failures where the circuits let the students know the train's response dynamically to their actions, in addition to understanding the causes behind the failure and how to detect and provide a solution to it. 
Moreover, failures and incidents, as well as the fire extinction system, can be studied by the students in a more convenient and flexible way.

Also, the high degree of simulation has become the beginning of a useful tool for ideas different from those sought.

Now, the simulator can be used not only for the training of drivers but for signalmen, engineers and maintenance personal. Each of them need training in different ways, but can find an opportunity for this in the Full Simulator.

In addition, the development of a simulator with different built-in systems has permitted not only single training, but also joint training of personnel from different areas. This process means that all of them perceive the problems of the work done by the others, and increases the training quality, giving personnel new extra knowledge.

On the negative side of the project's development is the long, hard work caused by the available information. The model of both trains, as well as the majority of the scenarios, were designed before the real ones. This means that the information available is not always in keeping with the end result, and converts the project into a system that is being continuously re-fed with new information that replaces the previous information.

The end result is that the project's real central issue has been documentation, which means that the information flow between all the parties involved must be as direct and precise as possible in order to avoid wasted effort in the development process.

Finally, it must be pointed out that the concept of a Training Centre managed by an instructor enables the efforts of training personnel to be maximized, leading to greater interaction between the persons involved in a training course. This, in turn, means a need for less direct supervision, which, however, must be compensated for by careful planning of the students' training plan.

\section{References}

[1] Simulation's definition at Wikipedia (http://en.wikipedia.org/wiki/ simulator)

[2] A Tour of the Amtrak Training Facility (http://www.weaverling.org/ atc/sim)

[3] BEVAC Consulting Engineers (Train simulator for Moroccan National Railways) (http://www.bevac.be/simulator.htm)

[4] CFF Re460 simulator - Swiss railways Loc training simulator (in French) (http://home.urbanet.ch/urba1206/Trains/files/simu.htm)

[5] CFL 3000 - A locomotive simulator in Luxembourg (http://www.rail.lu/ cflsimulateur.html)

[6] Eurostar Train simulator - A training simulator for Channel tunnel train drivers (http://www.qnx.com/company/customer_stories/ss_206_2.html)

[7] INCERTRANS- various original old training simulators (Romania) (http://www.afer.ro/rom/SIUSI_Simulatoare_vehicule.htm)

[8] PZL Loc simulator- Railway simulator by the Polish company PZL. (http://www.ai.com.pl/en/products/ep09_ds.html) 
[9] Simulator för spårburen trafik (SST) Swedish Simulator for Trackbased vehicles (http://www.it.uu.se/research/project/sst/api)

[10] Euskosim - Lander simulación (http://www.landersimulation.com/ index.php?id=29)

[11] Rotterdamse Metro (http://www.retmetro.nl/nl_msts_index.htm)

[12] Metro Bilbao - Simulator (http://www.metrobilbao.net/accesible/eng/ metro/simulador.html)

[13] Corys - Various Training simulators (http://www.corys.com/domaines/ railway-simulation.php)

[14] EADS - Train simulators (http://www.railway-technology.com/ contractors/professional/dornier)

[15] Krauss-Maffei Wegmann- Training simulators for military and civil vehicles (http://www.kmweg.com/gb/frame.php?page=44)

[16] Indra sistemas - Simuladores ferroviarios (http://www.indra.es/servlet/ ContentServer?pagename=IndraES/Proyecto_FA/DetalleProyecto\&cid=1 083830070898\&pid $=1083830064940 \&$ Language $=$ es_ES\&opc $=$ Listado)

[17] FAAC Incorporated (http://www.faac.com/railsimulators.htm)

[18] ORTHSTAR (http://www.orthstar.com/modsim4.htm)

[19] Visual simulation at Valencia University (Visor de línea 5) (http://robotica.uv.es/castellano/ARTEC/home.html\#Simulacion_civil)

[20] Onyx Real-Time Image Generation (http://www.futuretech.blinkenlights. nl/onyxrtig.html) 